\title{
Prioridades de requisitos para projeto de postos de operação de tratores quanto à ergonomia e segurança
}

\author{
Paula Machado dos Santos ${ }^{(1)}$, José Fernando Schlosser ${ }^{(1)}$, Leonardo Nabaes Romano(1), \\ Dinorvan Rozin ${ }^{(2)}$, Jeferson da Costa Turatti ${ }^{(1)}$ e Maiquel Witter ${ }^{(1)}$
}

\begin{abstract}
(1)Universidade Federal de Santa Maria, Avenida Roraima, № 1.000, Cidade Universitária, Bairro Camobi, CEP 97105-900 Santa Maria, RS. E-mail: paula.san@pop.com.br, schlosse@samil.ufsm.br, romano@smail.ufsm.br, gefersongct@yahoo.com.br, maiquelwitter@yahoo.com.br (2) John Deere Company do Brasil, Unidade de Horizontina, CEP 98920-000 Horizontina, RS. E-mail: rozindinorvan@johndeere.com
\end{abstract}

\begin{abstract}
Resumo - O objetivo deste trabalho foi identificar as prioridades de requisitos de projeto, de postos de operação de tratores agrícolas do mercado nacional brasileiro, conforme as normas de ergonomia e segurança. Compuseram a amostragem os tratores agrícolas de pneus de borracha novos, com bitola externa superior a $1.150 \mathrm{~mm}$, sem quaisquer alterações em suas características originais por parte do fabricante ou revendedor, em comercialização em território nacional, quanto à disposição interna dos comandos de operação, aos assentos e aos acessos dos postos de operação. Como análise comparativa dos requisitos dos clientes destas máquinas e, conseqüentemente, a hierarquização dos requisitos necessários ao projeto de postos de operação, que são atendidos pelos fabricantes de tratores agrícolas, foram utilizadas as ferramentas para o desenvolvimento de projetos diagrama de Mudge e a primeira matriz do "quality function deployment", respectivamente. As marcas de tratores agrícolas nacionais apresentam a mesma prioridade quanto ao atendimento aos requisitos de projeto de ergonomia e segurança em postos de operação. $\mathrm{O}$ assento representa a prioridade de maior ordem, seguido dos comandos e, em última posição, os acessos.
\end{abstract}

Termos para indexação: conforto do operador, desdobramento da função qualidade, diagrama de Mudge, requisitos do cliente.

\section{Requirement priorities for the design of tractor workplaces related to ergonomics and safety}

\begin{abstract}
The objective of this work was to characterize the requirement priorities for workplace design of agricultural tractors of the national market, as per standards of ergonomics and safety. The patterns were composed by agricultural new tractors rubber tires, with minimum gauge of $1,150 \mathrm{~mm}$, without any alterations in their original characteristics on the part of the manufacturer or reselling, in commercialization in national territory, related to the internal disposition of the operation commands, to the seats and to the accesses of the workplaces. As comparative analysis of the customer requirements for of these machines and, consequently, the hierarchization of the necessary requirements for the project of workplaces which are assisted by the manufacturers of agricultural tractors, the tools for the development of design Mudge diagram and the first head office of Quality Function Deployment where used. The marks of national agricultural tractors present the same priority related to requirements for ergonomics and safety designs in of workplace operation. The seat represent a larger order priority, followed by commands and, in the last position, the accesses.
\end{abstract}

Index terms: operator comfort, quality function deployment, diagram of Mudge, customer requirements.

\section{Introdução}

A preocupação com o conforto e a segurança do operador de tratores agrícolas tem chamado a atenção de profissionais de diversas áreas crescentemente, no sentido de se considerarem os fatores humanos (ergonomia) na concepção projetual de tratores agrícolas, em razão das adversidades impostas pela natureza no meio agrícola e, também, da periculosidade que essas máquinas apresentam e dos acidentes envolvidos nesse contexto (Santos Filho et al., 2003; Rozin, 2004). As principais causas dos acidentes com tratores agrícolas estão relacionadas indiretamente à ergonomia dos postos de operação e seus acessos, que podem dar origem às falhas humanas. 
$\mathrm{O}$ acesso à determinada máquina, bem como o conforto térmico, o campo visual, o esforço para acionamento dos comandos e as dimensões do posto do operador são aspectos importantes a serem observados, em uma avaliação ergonômica de máquinas, com vistas ao maior conforto, segurança e produtividade, durante a realização da jornada de trabalho (Fontana et al., 2004; Lima et al., 2005).

As normas internacionais ISO 4252 (ISO, 1992), ISO 4253 (ISO, 1993) e ISO 4254-1(ISO, 2008) e suas respectivas versões brasileiras NBR ISO 4252 (ABNT, 2000) NBR ISO 4254-1 (ABNT, 1999a) estabelecem dimensões para o acesso aos postos de operação (degraus e estribos), bem como para o espaço de movimentação interna. Os degraus devem ser projetados e posicionados de forma a não serem atingidos e danificados, durante a operação da máquina, e ainda para evitar que se tornem causa de inúmeros acidentes, principalmente quando não se dispõe de estribos e corrimões projetados corretamente para o uso do tratorista (Robin, 1987; Schlosser \& Debiasi, 2002).

Quando se trata da comodidade de se trabalhar em um posto de operação, o assento tem sido muito utilizado como palavra-chave. Constatado o surgimento de doenças e enfermidades vinculadas ao trabalho sedentário, passou-se a considerar o fator ergonomia como essencial nos projetos de assentos, em conjunto com o ambiente de trabalho. Quanto às características que o assento do operador deve possuir, destacam-se as dimensões (Debiasi et al., 2004). A ISO 4253 (ISO, 1993) padroniza dimensões para o projeto de assento do operador de tratores agrícolas, como a sua altura em relação à plataforma de apoio para os pés, o comprimento do assento em relação ao ponto de indexação do assento (SIP), as larguras da almofada do assento e do encosto lombar, e o comprimento do encosto do assento.

Schlosser et al. (2002) destacam a importância da possibilidade de o operador alcançar e acionar, com o mínimo esforço e de forma a manter postura corporal correta, todos os comandos - volante de direção, pedais dos freios e da embreagem, acelerador, chaves de comandos, entre outros -, que devem estar dispostos e montados sobre o posto de operação do trator agrícola, de maneira a permitir o controle, com manuseio fácil e seguro pelo seu operador, na sua posição normal de trabalho. Deve-se conhecer o espaço, que as mãos e os braços necessitam para a preensão e movimento, e que passa a ser uma importante premissa para o planejamento de controles, comandos e demais ferramentas do local de trabalho, explica Grandjean (1998). Os comandos devem apresentar uma série de características definidas por normas - como pela ISO 15077 (ISO, 1996) que delimita áreas de alcance para o operador sentado, em relação ao mesmo ponto de referência - que abrangem todos os comandos de operação no posto de operação de máquinas agrícolas autopropelidas.

O objetivo deste trabalho foi caracterizar as prioridades de requisitos, de projetos de postos de operação de tratores agrícolas do mercado nacional brasileiro, por meio de suas conformidades às especificações padronizadas por normas de ergonomia e segurança.

\section{Material e Métodos}

Este trabalho foi realizado no Núcleo de Ensaio de Máquinas Agrícolas (Nema) da Universidade Federal de Santa Maria, Santa Maria, RS. Foram amostrados postos de operação de tratores agrícolas com pneus de borracha novos, com bitola externa superior a $1.150 \mathrm{~mm}$, com todas as marcas de tratores agrícolas em comercialização no mercado brasileiro. Os tratores agrícolas foram divididos em classes de potência bruta no motor, e agrupados de acordo com os seus postos de operação. Este agrupamento considerou postos de operação homogêneos, identificados de acordo com os seguintes parâmetros: tipo de posto de operação (acavalado ou plataformado); assento do operador; versão e disposição interna dos comandos de operação. Assim, um posto de operação pôde abranger um ou mais modelos de tratores agrícolas.

A amostragem foi executada mediante visitas às concessionárias autorizadas de tratores agrícolas. Consideraram-se os padrões ergonômico e de segurança nos tratores agrícolas estabelecidos pelas fábricas. Uma equipe de trabalho, composta por três pessoas, ficava responsável pela aquisição das medidas ergonométricas dos postos de operação dos tratores, em relação às dimensões do acesso ao posto de operação em cada trator amostrado e ao SIP, definido pela norma NBR/NM/ISO 5353 (ABNT, 1999b), como um ponto situado no plano médio longitudinal central do assento, onde o plano tangencial do encosto intersecciona um plano horizontal, equivalente à intersecção do plano 
vertical, que contém a linha central do assento no eixo teórico entre o tronco humano e as coxas.

Foram amostrados postos de operação de tratores agrícolas das empresas que atuam no mercado brasileiro, identificadas pelas marcas: Agrale, Case, John Deere, Massey-Ferguson, New Holland, Valtra e Yanmar. Cada posto de operação homogêneo recebeu uma denominação para melhor compreensão, descrita na Tabela 1.

Por meio de revisão de literatura, com citações sobre as principais necessidades de operadores de tratores, para preservar sua segurança e atender à sua ergonomia, as características de postos de operação de tratores agrícolas foram definidas como requisitos dos clientes, comparadas entre si com o objetivo de se determinar a importância relativa entre elas, conforme proposta de Mudge (Márquez, 1990; Kumar et al., 1998; Debiasi, 2002; Valent et al., 2002). Pelo diagrama de Mudge (Csillag, 1995) faz-se a análise numérica funcional, que prioriza as funções por ordem de importância que elas representam para o cumprimento das exigências requeridas pelos usuários. Esse método compara as funções em pares, pelo grau de importância que uma apresenta em relação à outra. Esses valores podem

Tabela 1. Denominações dos postos de operação (PO) considerados homogêneos, pelas características de: tipo (acavalado ou plataformado), assento do operador, versão e disposição interna dos comandos de operação.

\begin{tabular}{|c|c|c|}
\hline $\mathrm{PO}^{(1)}$ & Características do $\mathrm{PO}^{(2)}$ & Modelos de tratores correspondentes \\
\hline $1 \mathrm{~A}$ & Acavalado, padrão & AG 4230 e $A G 4240$ \\
\hline $1 \mathrm{~B}$ & Plataformado, padrão, sem cabine & 5075.4 e 5085.4 \\
\hline $1 \mathrm{C}$ & Plataformado, padrão, sem cabine & BX6150 \\
\hline $2 \mathrm{~A}$ & Plataformado, com cabine & MXM135, MXM150 e MXM165 \\
\hline $3 \mathrm{~A}$ & Acavalado, padrão, sem cabine & 5403 \\
\hline 3B & Plataformado, padrão, sem cabine & 5605 e 5705 \\
\hline $3 \mathrm{C}$ & Plataformado, padrão, sem cabine & 6405,6605 e 7505 \\
\hline $3 \mathrm{D}$ & Plataformado, padrão, com cabine & 6405,6605 e 7505 \\
\hline$\overline{4 \mathrm{~A}}$ & Acavalado, padrão, sem cabine & MF265, MF275 e MF283 \\
\hline $4 \mathrm{~B}$ & Acavalado, versão "trator longo", sem cabine & MF265, MF275 e MF283 \\
\hline $4 \mathrm{C}$ & Acavalado, padrão, sem cabine & MF290, MF292, MF297 e MF299 \\
\hline 4D & Plataformado, com cabine & $\begin{array}{l}\text { MF275, MF283, MF290, MF292, MF297, MF299, } \\
\text { MF5275, MF5285 e MF5290 }\end{array}$ \\
\hline $4 \mathrm{E}$ & Plataformado, sem cabine & MF5275, MF5285 e MF5290 \\
\hline $4 \mathrm{~F}$ & Plataformado, sem cabine & MF5310 e MF5320 \\
\hline $4 \mathrm{G}$ & Plataformado, padrão, sem cabine & MF650, MF660 e MF680 \\
\hline$\underline{4 \mathrm{H}}$ & Plataformado, padrão, com cabine & MF5310, MF5320, MF650, MF660 e MF680 \\
\hline$\overline{5 \mathrm{~A}}$ & Plataformado, padrão, sem cabine & TL55E, TL65E, TL75E, TL85E e TL 95E \\
\hline $5 \mathrm{~B}$ & Plataformado, padrão, sem cabine & TL70 TL80, TL90 e TL100 \\
\hline $5 \mathrm{C}$ & Acavalado, padrão, sem cabine & 7630 e 8030 \\
\hline $5 \mathrm{D}$ & $\begin{array}{l}\text { Plataformado, padrão, sem cabine, com transmissão do tipo não sincronizada (Econoshift } \\
16 \mathrm{X} 4 \text { ), que é o fator diferencial em comparação aos postos de operação } 5 \mathrm{E} \text { e } 5 \mathrm{~F}\end{array}$ & TS100, TS110 e TS120 \\
\hline $5 \mathrm{E}$ & $\begin{array}{l}\text { Plataformado, padrão, sem cabine, com transmissão do tipo sincronizada (Synchro } \\
\text { Command 12X12) }\end{array}$ & TS100, TS110 e TS120 \\
\hline $5 \mathrm{~F}$ & $\begin{array}{l}\text { Plataformado, padrão, com cabine, com transmissão do tipo sincronizada (Synchro } \\
\text { Command } 12 \mathrm{X} 12 \text { ), que é o fator diferencial em comparação ao posto de operação } 5 \mathrm{E}\end{array}$ & TS100, TS110 e TS120 \\
\hline $5 \mathrm{G}$ & $\begin{array}{l}\text { Plataformado, padrão, sem cabine, com transmissão do tipo sincronizada (Shuttle } \\
\text { Command 16X16) }\end{array}$ & TM135, TM150 e TM165 \\
\hline $5 \mathrm{H}$ & $\begin{array}{l}\text { Plataformado, padrão, sem cabine, com transmissão do tipo sincronizada (Dual Command } \\
24 \times 12 \text { ), que é o fator diferencial nesse posto de operação em comparação ao posto } 5 \mathrm{G}\end{array}$ & TM135, TM150 e TM165 \\
\hline $5 \mathrm{I}$ & $\begin{array}{l}\text { Plataformado, padrão, com cabine, com transmissão do tipo “power shift” (Range } \\
\text { Command 31X24) }\end{array}$ & TM135, TM150 e TM165 \\
\hline $6 \mathrm{~A}$ & Acavalado, padrão, sem cabine & BF65 e BF75 \\
\hline $6 \mathrm{~B}$ & Acavalado, padrão, sem cabine & 685 e 785 \\
\hline $6 \mathrm{C}$ & Plataformado, padrão, sem cabine & 700,800 e 900 \\
\hline $6 \mathrm{D}$ & Plataformado, padrão, sem cabine & 985, BM85, BM100, BM110 e BM120 \\
\hline $6 \mathrm{E}$ & $\begin{array}{l}\text { Plataformado, padrão, com ou sem cabine, exceto para a cabine do tipo "ray comfort" que } \\
\text { pode equipar os modelos com prefixo BH }\end{array}$ & 1280R, 1580, 1780, ВН140, ВН160 е ВН180 \\
\hline $7 \mathrm{~A}$ & $\begin{array}{l}\text { Acavalado, padrão, sem cabine, equipados com assento-padrão "baixo", que não apresenta } \\
\text { regulagens ou ajustes }\end{array}$ & 1045 e $1045 \mathrm{DT}$ \\
\hline
\end{tabular}

(1)1, Agrale; 2, Case; 3, John Deere; 4, Massey Ferguson; 5, New Holland; 6, Valtra; 7, Yanmar; cada letra significa um grupo de tratores com características homogêneas, de acordo com parâmetros do tipo do posto de operação, do assento do operador, da influência da versão e da disposição interna dos comandos. (2) Acavalado: postos de operação formados por estribos colocados lateralmente ao monobloco do trator; plataformado: a superfície de apoio para os pés coincide com um plano próximo da horizontal, e é montada sobre o monobloco ou chassi do trator; padrão: trator agrícola oferecido pelo fabricante com suas características básicas de conforto e segurança ao operador. 
caracterizar um nível de importância entre os requisitos de clientes tanto para a projeção de postos de operação, quanto para a aquisição de determinados tratores no mercado. Caracterizaram-se como os principais requisitos dos clientes, quanto à funcionalidade de elementos presentes em postos de operação: órgãos de comando distribuídos adequadamente (1), a comodidade dos assentos (2), acessos projetados adequadamente (3) e, ainda, foi considerada a presença de espaços e movimentação interna inadequados (4), requisitos estes submetidos ao diagrama de Mudge, para se determinar sua valoração (Tabela 2). Para cada requisito do cliente, foi atribuído um grau de importância em ordem decrescente, representado da seguinte maneira: $\mathrm{A}=5$; $\mathrm{B}=3 ; \mathrm{C}=1$.

A aplicação do diagrama Mudge demonstra que embora todas as características sejam necessárias, algumas apresentam grau de importância diferenciado entre os operadores de tratores agrícolas. Neste caso, a presença de espaços e movimentação interna inadequados não apresentou importância relativa quanto aos demais requisitos de projeto, não foi assim, comparada com os demais requisitos de projeto. Buscouse estabelecer requisitos de projeto pela proposição de normas realizadas pela International Organization for Standartization (ISO) e pela sua versão brasileira NBR ISO, que são descritas a seguir.

Conforme as normas NBR ISO 4254-1 (ABNT, 1999a) e NBR ISO 4252 (ABNT, 2000), a altura do primeiro degrau, em relação ao solo, não deverá ultrapassar $550 \mathrm{~mm}$, em que a dimensão ideal é de $500 \mathrm{~mm}$. A profundidade e a largura dos degraus deverão ser no mínimo de 150 e $200 \mathrm{~mm}$, respectivamente. A distância vertical do último degrau até a soleira da plataforma do trator deverá ser no

Tabela 2. Determinação dos requisitos de clientes relativos à ergonomia e segurança de operadores de postos de operação de tratores agrícolas (método de Mudge) ${ }^{(1)}$.

\begin{tabular}{|c|c|c|c|c|c|}
\hline \multicolumn{4}{|c|}{ Requisitos } & \multirow{2}{*}{$\begin{array}{l}\text { Número de } \\
\text { pontos }\end{array}$} & \multirow{2}{*}{$\begin{array}{c}\text { Importância } \\
(\%)\end{array}$} \\
\hline & 2 & 3 & 4 & & \\
\hline \multirow[t]{4}{*}{1} & $1 \mathrm{~B}^{(2)}$ & $1 \mathrm{~B}$ & $1 \mathrm{~A}$ & 11 & 70,00 \\
\hline & 2 & $2 \mathrm{C}$ & $2 \mathrm{~B}$ & 4 & 25,00 \\
\hline & & 3 & $3 \mathrm{C}$ & 1 & 0,05 \\
\hline & & & 4 & 0 & 0,00 \\
\hline Total & & & & 21 & 100,00 \\
\hline
\end{tabular}

(1) 1 = órgãos de comandos distribuídos adequadamente; 2 = comodidade dos assentos; 3 = acessibilidade dos degraus projetados adequadamente; 4 = presença de espaços e movimentação interna adequados.

${ }^{(2)} \mathrm{A}=5$, maior grau de importância; $\mathrm{B}=3$, médio grau de importância; $\mathrm{C}=1$, menor grau de importância. máximo de $300 \mathrm{~mm}$, para os acessos na configuração multidegraus, e de $350 \mathrm{~mm}$ para o acesso na configuração monodegraus. A distância vertical entre degraus deve ser no máximo de $300 \mathrm{~mm}$, segundo a mesma norma de referência.

A norma ISO 4253 (ISO, 1993) padroniza dimensões para projeto do assento que são: a altura do assento em relação à plataforma de apoio para os pés, que deve ser de $450 \mathrm{~mm}$ no mínimo e $520 \mathrm{~mm}$ no máximo; o comprimento do assento em relação ao SIP, que deve ser de $260 \mathrm{~mm}$ (ótimo), ou seja, ótimo por ser considerado padrão entre operadores, de acordo com suas medidas antropométricas, que podem variar $50 \mathrm{~mm}$ para mais ou para menos; a largura da almofada do assento e a largura do encosto lombar devem ser superiores a $450 \mathrm{~mm}$; e o comprimento do encosto do assento deve ser no mínimo de $260 \mathrm{~mm}$.

Todos os comandos de operação, no posto de operação de máquinas agrícolas autopropelidas, são determinados por meio da norma ISO 15077 (ISO, 1996), que delimita áreas de alcance para o operador sentado, em relação ao mesmo ponto de referência, e estabelece as zonas de conforto, de acesso e as inacessíveis, de acordo com a frequiência de utilização desses comandos. A zona de conforto permite fácil acesso aos comandos pelo operador; nela devem estar situados os comandos principais acionados pelas mãos e pés. A zona de acesso permite um alcance do condutor do trator agrícola desde a sua posição sentada, pois admite deslocamento e inclinação lateral ou adiante para acionar comandos de operação nessa posição. Um comando localizado numa zona inacessível ao alcance do operador ocorrerá, quando estiver localizado fora da zona de conforto ou de acesso definidas anteriormente.

Para se obter o grau de importância, utilizou-se a construção de uma matriz de correlação entre os requisitos de cliente e os requisitos de projeto. Para obtenção da matriz de correlação, apresentada na Tabela 3, buscou-se verificar, para cada requisito de cliente, sua contribuição para a incorporação de diferentes requisitos de projeto no produto final (postos de operação). Se a correlação existir, identifica-se simbolicamente o grau desta correlação com a seguinte simbologia: $\otimes$, forte correlação (peso 9 ); $\bigcirc$, moderada correlação (peso 3); e $\Delta$, fraca correlação (peso 1). A seguir, multiplica-se o peso da correlação entre atividades pelo grau de importância da função obtida pelo diagrama de Mudge. Somando-se os valores obtidos 
nas diversas correlações, para uma mesma atividade (coluna da matriz), tem-se o grau de importância de todas as atividades pertencentes ao processo. Utilizou-se a ferramenta de projeto matriz do desdobramento da função qualidade, ou primeira matriz do QFD ("quality function deployment"), para identificar a priorização dos requisitos de projeto de postos de operação, de cada marca e modelo de tratores nacionais (Romano, 2003).

A partir de cada dimensão estabelecida pelas normas ISO 15077 (ISO, 1996) e NBR ISO 4254-1 (ABNT, 1999a), foram elaboradas faixas com valores limites, tendo-se partido de cada valor citado como o mínimo ou máximo da especificação, considerados parcialmente conformes, e consideraram-se em conformidade plena com a norma os valores até $10 \%$ maiores ou menores que seus limites para conformidade (Tabela 4).

Os órgãos de comando de cada posto de operação foram considerados conformes, parcialmente conformes e não conformes, quando o mínimo de 80 e $50 \%$ e o máximo de $49 \%$, respectivamente, estavam dentro da zona de conforto.

\section{Resultados e Discussão}

As prioridades no atendimento aos requisitos de projeto, quanto à ergonomia e segurança estabelecidas pelas normas

Tabela 3. Graus de importância (GI) dos requisitos de cliente, associados aos requisitos de projeto, correspondentes às regiões de alcance dos comandos (CO), dimensões do assento do operador (AST) e dimensões do acesso (ACS) de postos de operação (PO), dos tratores agrícolas Case 5075 e 5085 (modelo QFD - “quality function deployment”) $)^{(1)}$.

\begin{tabular}{|c|c|c|c|c|c|c|c|c|c|c|c|c|c|c|c|c|}
\hline \multirow[t]{2}{*}{$\overline{\mathrm{PO}^{(2)}}$} & \multirow[t]{2}{*}{ GI } & \multirow{2}{*}{$\frac{\mathrm{CO}}{\mathrm{ZC}}$} & \multicolumn{9}{|c|}{ AST } & \multicolumn{5}{|c|}{ ACS } \\
\hline & & & ARP & CRP & LA & LE & $\mathrm{CE}$ & $\mathrm{AL}$ & AVPM & RIE & $\overline{R P}$ & APD & PD & LD & DVUDS & EED \\
\hline 1 & 70 & $3 \backslash 0$ & & & & & & & & & & & & & & \\
\hline 2 & 25 & - & $1 \backslash \triangle$ & 310 & $3 \backslash^{\circ}$ & 310 & $3 \backslash 0$ & $3 \backslash 0$ & 3) & $1 \backslash \Delta$ & $1 \backslash \Delta$ & & & & & \\
\hline 3 & 5 & & & & & & & & & & & $1 \triangle$ & $3 \backslash 0$ & 310 & $1 \backslash \triangle$ & $M^{(3)}$ \\
\hline Total & 100 & 210 & 25 & 75 & 75 & 75 & 75 & 75 & 75 & 25 & 25 & 5 & 15 & 15 & 5 & - \\
\hline GI (\%) & & 27,1 & 3,23 & 9,68 & 9,68 & 9,68 & 9,68 & 9,68 & 9,68 & 3,23 & 3,23 & 0,65 & 1,94 & 1,94 & 0,65 & - \\
\hline
\end{tabular}

${ }^{(1)}$ ZC: zona de conforto; ARP: altura em relação à plataforma; CRSIP: comprimento em relação ao ponto de inclinação do assento (SIP); LA: largura da almofada; LE: largura do encosto; CE: comprimento do encosto; AL: ajuste longitudinal; AVPM: ajuste vertical a partir da posição média; RIE: regulagem da inclinação do encosto; RP: regulagem de pressão; APD: altura do primeiro degrau; PD: profundidade do degrau; LD: largura do degrau; DVUDS: distância vertical do último degrau até a soleira; EED: espaçamento entre degraus; O, moderada correlação (peso 3 ); $\Delta$, fraca correlação (peso 1). ${ }^{(2)}$ 1, órgãos de comando distribuídos adequadamente; 2, comodidade dos assentos; 3 , acessos projetados adequadamente. ${ }^{(3)}$ Monodegrau.

Tabela 4. Delimitações para dimensões de acessos e assentos de postos de operação de tratores agrícolas, segundo a conformidade às normas ISO 15077 (ISO, 1996), ISO 4253 (ISO, 1993) e NBR ISO 4254-1 (ABNT, 1999a).

\begin{tabular}{|c|c|c|c|c|}
\hline \multirow[t]{2}{*}{ Especificação } & \multirow{2}{*}{$\begin{array}{l}\text { ISO, NBR ISO } \\
\text { (dimensões em mm) }\end{array}$} & \multicolumn{3}{|c|}{ Comparativo $(\mathrm{mm})^{(1)}$} \\
\hline & & $\mathrm{C}$ & $\mathrm{CP}$ & $\mathrm{NC}$ \\
\hline Largura dos degraus & 200 (mínimo) & 220 & $\geq 200$ & $<200$ \\
\hline Profundidade dos degraus & 150 (mínimo) & 165 & $\geq 150$ & $<150$ \\
\hline Distância vertical entre os degraus & 300 (máximo) & 270 & $\leq 300$ & $>300$ \\
\hline $\begin{array}{l}\text { Distância vertical do último degrau à soleira da plataforma } \\
\text { (multidegraus) }\end{array}$ & 300 (máximo) & 270 & $\leq 300$ & $>300$ \\
\hline $\begin{array}{l}\text { Distância vertical do último degrau à soleira da plataforma } \\
\text { (monodegraus) }\end{array}$ & 350 (máximo) & 315 & $\leq 350$ & $>350$ \\
\hline Altura do primeiro degrau em relação ao solo & 550 (máximo) & 495 & $\leq 550$ & $>550$ \\
\hline Ajuste longitudinal do assento a partir da posição média & $\begin{array}{l}75 \text { (mínimo) } \\
100 \text { (ótimo) }\end{array}$ & 100 & $\geq 75$ & $<75$ \\
\hline Ajuste vertical do assento a partir da posição média & $\begin{array}{l}30 \text { (mínimo) } \\
50 \text { (ótimo) }\end{array}$ & 50 & $\geq 30$ & $<30$ \\
\hline $\begin{array}{l}\text { Comprimento do assento em relação ao ponto de indexação } \\
\text { o assento }\end{array}$ & $260 \pm 50$ & 260 & $210-310$ & $\begin{array}{l}<210 \\
>310\end{array}$ \\
\hline Largura total do assento (almofada) & 450 (mínimo) & 495 & $\geq 450$ & $<450$ \\
\hline Comprimento total do encosto lombar do assento & 260 (mínimo) & $\geq 260$ & - & $<260$ \\
\hline Largura total do encosto lombar do assento & 450 (mínimo) & 495 & $\geq 450$ & $<450$ \\
\hline $\begin{array}{l}\text { Altura do ponto de indexação do assento, em relação à } \\
\text { Plataforma de apoio para os pés }\end{array}$ & $485 \pm 35$ & 485 & $520-450$ & $\begin{array}{l}>520 \\
<450\end{array}$ \\
\hline Ajuste vertical a partir da posição média & $\begin{array}{l}75 \text { (mínimo) } \\
50 \text { (ótimo) }\end{array}$ & & & \\
\hline
\end{tabular}

${ }^{(1)} \mathrm{C}$ : confomidade; $\mathrm{CP}$ : conformidade parcial; NC: não conforme. 
ISO 15077 (ISO, 1996), ISO 4253 (ISO, 1993) e NBR ISO 4254-1 (ABNT, 1999a), correspondentes às regiões de alcance dos comandos, dimensões do assento do operador e dimensões do acesso do posto de operação, respectivamente, estão demonstradas na Tabela 5. Os números ordinais em ordem decrescente representam o nível de importância, reportado para cada requisito de projeto, para cada tipo de postos de operação.

Os valores percentuais indicam atendimento às normas dos postos de operação dos tratores avaliados, que atendem à conformidade das normas para cada critério de projeto analisado, independentemente de ser plena ou parcialmente.

Quanto ao atendimento às conformidades, em todos os postos de operação avaliados, os critérios de projeto referentes aos comandos de operação e às medidas do assento do operador demonstraram estar como as principais prioridades, segundo Rozin (2004), que verificou não haver relação direta entre a magnitude de potência bruta do motor e os comandos melhor posicionados ergonomicamente em seus postos de operação.

A maioria das dimensões de cada critério não apresentou conformidade plena e sim valores próximos às disposições de conformidade.

A ISO 4253 (ISO, 1993) não estabelece um valor máximo, como limite para o comprimento do encosto, e pode gerar confusões durante a decisão sobre qual assento teria o melhor encosto. Grandjean (1998) cita que o encosto do assento possui a função de apoiar as costas do operador, principalmente entre a área do sacro e a região lombar, porém um encosto lombar muito elevado pode limitar os movimentos das costas e dos ombros. Debiasi (2002) afirma que o encosto deve suportar parte do peso correspondente à cabeça e aos membros superiores, para ajudar a reduzir o trabalho estático a que se encontram submetidos os músculos das costas e ainda, reduzir a pressão exercida sobre os discos intervertebrais.

Observa-se na Tabela 5 que o critério altura do SIP, em relação à plataforma, foi atendida por apenas $16,1 \%$ dos postos de operação. Segundo Mehta \& Tewari (2000), este critério representa a medida de maior importância para o assento. Para que exista maior integração entre a superfície de apoio para os pés e a superfície de trabalho (plataforma), o assento deve possuir mecanismos de regulagem de altura. Grandjean (1998) destaca valores mínimos e máximos de 380 e $500 \mathrm{~mm}$, respectivamente, para a altura do assento.
Apenas os tratores MXM135, MXM150 e MXM165 apresentam regulagens verticais, o que compreende apenas 3,2\% do total de postos de operação analisados. Esta regulagem é importante, pois permite que o assento gire em torno do seu eixo vertical e facilite a mobilização do operador, reduzindo problemas musculares no pescoço e ombros.

Os tratores Agrale BX6150, New Holland modelos 7630,8030, TL70, TL80, TL90 e TL100, Valmet modelos 700,800 e 900 priorizaram em seus projetos de postos de operação a disposição de comandos dentro da zona de conforto, para reduzir a ocorrência de movimentos secundários do tronco, causadores de riscos de problemas nas costas e ombros e, para aumentar a segurança da operação (Couto, 1996; Grandjean, 1998). De acordo com a norma ISO 15077 (ISO, 1996), a utilização de comandos dentro da zona de conforto permite o fácil acesso pelo operador e, nela, devem estar situados os comandos de pé e de mãos freqüentemente utilizados. Os demais tratores apresentaram conformidade parcial quanto à disposição de seus comandos na área de acesso, que exigem deslocamento e inclinação lateral ou adiante, para acionar os comandos de operação e, assim, atendem secundariamente aos requisitos dos clientes, que julgaram ser este critério mais importante quanto à ergonomia e segurança de um posto de operação.

Os degraus apresentaram a menor prioridade, quanto ao atendimento dos critérios de projeto, principalmente porque todas as dimensões de degraus apresentaram conformidade parcial aos requisitos de projeto, na totalidade dos postos de operação avaliados, enquanto os demais critérios para assentos e comandos apresentaram, no mínimo, uma conformidade plena. Tratores cujo acesso ao posto de operação não está dentro das normas de segurança, ou seja, com degraus inexistentes ou mal localizados, sem superfície antiderrapante e estreitos, facilitam a ocorrência de escorregões e potencializam a ocorrência de quedas, o que resulta em ferimentos leves, tais como hematomas, cortes, luxações e torções (Schlosser, 2001). Um escorregão no momento em que o operador está subindo ao posto de trabalho do trator pode causar queda, apontada como um dos acidentes de maior incidência na Espanha, pois representa 30\% de todos os acidentes com tratores agrícolas (Márquez, 1990). Na região da Depressão Central do Rio Grande do Sul, o tipo de acidente leve, de ocorrência mais frequiente, foram os escorregões, com 40,82\%, seguidos das lesões em quinas 
agudas com $28,57 \%$ e queda do trator com $18,37 \%$ (Debiasi, 2002).

As dificuldades em se caracterizarem determinadas medidas do posto de operação como conformes ou parcialmente conformes ocorreram, pois as normas estabelecem apenas um valor considerado ótimo ou limite para o seu atendimento, no entanto, são poucas vezes estabelecidas as faixas que podem caracterizar o atendimento às normas. Assim, poderiam ocorrer possíveis conflitos entre os responsáveis pela projeção dessas características, principalmente, quando ocorresse $\mathrm{o}$ atendimento de determinado valor limite mínimo, porém, o não estabelecimento de um valor limite máximo estaria confrontado com os demais requisitos de projeto. Como exemplo, pode-se citar o atendimento o da medida da largura dos degraus, que deve ser maior a $200 \mathrm{~mm}$. No entanto, observou-se que, apesar de a maioria dos postos de operação cumprir determinado padrão, foram apresentadas larguras superiores à estabelecida pela norma, o que pode influenciar nas medidas de construção dos paralamas ou, ainda, nas demais seções de construção do projeto de um trator e, assim, as medidas seriam parcialmente conformes e não de conformidade plena, como estabelece a norma.

A equipe que visitou os revendedores de tratores verificou as informações ergonométricas relativas a comandos, acessos e assentos, descritos a seguir.

E entre os comandos de uso freqüente os comandos chave de partida e estrangulador foram os que estiveram mais bem posicionados nos postos de operação

Tabela 5. Prioridades no atendimento aos requisitos (1 a 5 são colocações) de projeto, quanto à ergonomia e segurança estabelecidas pelas normas ISO 15077 (ISO, 1996), ISO 4253 (ISO, 1993) e NBR ISO 4254-1 (ABNT, 1999a), correspondentes às regiões de alcance dos comandos (CO), dimensões do assento do operador (AST) e dimensões do acesso (ACS) do posto de operação $(\mathrm{PO})$ de tratores nacionais ${ }^{(1)}$.

\begin{tabular}{|c|c|c|c|c|c|c|c|c|c|c|c|c|c|c|c|}
\hline \multirow[t]{2}{*}{$\overline{\mathrm{PO}}$} & \multirow{2}{*}{$\frac{\mathrm{CO}}{\mathrm{ZC}}$} & \multicolumn{9}{|c|}{ AST } & \multicolumn{5}{|c|}{$\mathrm{ACS}$} \\
\hline & & $\overline{\mathrm{ARP}}$ & CRSIP & LA & LE & $\mathrm{CE}$ & $\mathrm{AL}$ & AVPM & RIE & RP & APD & PD & LD & DVUDS & EED \\
\hline$\overline{1 \mathrm{~A}}$ & 2 & 0 & 3 & 0 & 0 & 1 & 0 & 0 & 0 & 0 & - & - & - & - & - \\
\hline $1 \mathrm{~B}$ & 2 & 0 & 3 & 3 & 3 & 1 & 3 & 0 & 0 & 3 & 0 & 0 & 4 & 0 & $\mathrm{M}^{(2)}$ \\
\hline $1 \mathrm{C}$ & 1 & 0 & 3 & 3 & 3 & 2 & 3 & 0 & 0 & 3 & 0 & 0 & 4 & 5 & 5 \\
\hline $2 \mathrm{~A}$ & 2 & 0 & 3 & 3 & 0 & 1 & 3 & 3 & 3 & 3 & 4 & 0 & 4 & 4 & 4 \\
\hline $3 \mathrm{~A}$ & 2 & 0 & 3 & 3 & 0 & 1 & 3 & 0 & 0 & 3 & 0 & 0 & 4 & 0 & $\mathrm{M}$ \\
\hline $3 \mathrm{~B}$ & 2 & 0 & 3 & 3 & 0 & 1 & 0 & 0 & 0 & 3 & 0 & 4 & 4 & 4 & $\mathrm{M}$ \\
\hline $3 \mathrm{C}$ & 2 & 0 & 3 & 3 & 0 & 1 & 3 & 0 & 0 & 3 & 0 & 4 & 0 & 4 & $\mathrm{M}$ \\
\hline $3 \mathrm{D}$ & 2 & 0 & 3 & 3 & 0 & 1 & 3 & 0 & 3 & 3 & 0 & 4 & 4 & 0 & 4 \\
\hline $4 \mathrm{~A}$ & 2 & 0 & 3 & 3 & 0 & 1 & 3 & 0 & 0 & 3 & 0 & 0 & 0 & 4 & $\mathrm{M}$ \\
\hline $4 \mathrm{~B}$ & 2 & 0 & 3 & 3 & 0 & 1 & 3 & 0 & 0 & 3 & 0 & 0 & 0 & 4 & $\mathrm{M}$ \\
\hline $4 \mathrm{C}$ & 2 & 0 & 3 & 3 & 3 & 1 & 3 & 0 & 0 & 3 & 0 & 0 & 0 & 4 & $\mathrm{M}$ \\
\hline $4 \mathrm{D}$ & 2 & 3 & 3 & 3 & 3 & 1 & 3 & 0 & 0 & 3 & 0 & 0 & 4 & 0 & 4 \\
\hline $4 \mathrm{E}$ & 2 & 0 & 3 & 3 & 3 & 1 & 3 & 0 & 0 & 3 & 0 & 0 & 0 & 4 & $\mathrm{M}$ \\
\hline $4 \mathrm{~F}$ & 2 & 0 & 3 & 3 & 0 & 1 & 3 & 0 & 0 & 3 & 0 & 0 & 4 & 0 & $\mathrm{M}$ \\
\hline $4 \mathrm{G}$ & 2 & 0 & 3 & 3 & 0 & 1 & 3 & 0 & 0 & 3 & 0 & 0 & 4 & 4 & 4 \\
\hline $4 \mathrm{H}$ & 2 & 0 & 0 & 3 & 3 & 1 & 3 & 0 & 3 & 3 & 0 & 0 & 4 & 4 & 4 \\
\hline $5 \mathrm{~A}$ & 2 & 0 & 3 & 3 & 0 & 1 & 3 & 0 & 0 & 3 & 4 & 4 & 4 & 4 & 4 \\
\hline $5 B$ & 2 & 0 & 3 & 3 & 0 & 1 & 3 & 0 & 0 & 3 & 4 & 4 & 4 & 4 & 4 \\
\hline $5 \mathrm{C}$ & 1 & 3 & 3 & 3 & 0 & 2 & 0 & 0 & 0 & 3 & 0 & 4 & 4 & 4 & 4 \\
\hline $5 \mathrm{D}$ & 2 & 3 & 3 & 3 & 0 & 1 & 0 & 0 & 0 & 3 & 0 & 4 & 4 & 4 & 4 \\
\hline $5 \mathrm{E}$ & 2 & 3 & 3 & 3 & 0 & 1 & 3 & 0 & 0 & 3 & 0 & 4 & 4 & 4 & 4 \\
\hline $5 \mathrm{~F}$ & 2 & 0 & 3 & 3 & 3 & 1 & 3 & 0 & 0 & 3 & 0 & 4 & 4 & 4 & 4 \\
\hline $5 \mathrm{G}$ & 2 & 0 & 3 & 3 & 3 & 1 & 3 & 0 & 0 & 3 & 4 & 4 & 4 & 4 & 4 \\
\hline $5 \mathrm{H}$ & 2 & 0 & 3 & 3 & 3 & 1 & 3 & 0 & 0 & 3 & 4 & 4 & 4 & 4 & 4 \\
\hline $5 \mathrm{I}$ & 2 & 3 & 0 & 3 & 3 & 1 & 3 & 0 & 3 & 3 & 4 & 4 & 4 & 4 & 4 \\
\hline $6 \mathrm{~A}$ & 2 & 0 & 3 & 3 & 0 & 1 & 0 & 0 & 0 & 3 & 4 & 4 & 4 & 4 & $\mathrm{M}$ \\
\hline $6 \mathrm{~B}$ & 1 & 0 & 3 & 3 & 0 & 2 & 0 & 0 & 0 & 3 & 0 & 0 & 0 & 4 & $\mathrm{M}$ \\
\hline $6 \mathrm{C}$ & 1 & 0 & 3 & 3 & 3 & 2 & 3 & 0 & 0 & 3 & 4 & 4 & 4 & 4 & 4 \\
\hline $6 \mathrm{D}$ & 2 & 0 & 3 & 3 & 0 & 1 & 3 & 0 & 0 & 3 & 0 & 4 & 4 & 4 & 4 \\
\hline $6 \mathrm{E}$ & 2 & 0 & 3 & 3 & 3 & 1 & 3 & 0 & 0 & 3 & 0 & 4 & 4 & 4 & $\mathrm{M}$ \\
\hline 7A & 2 & 0 & 3 & 3 & 0 & 1 & 0 & 0 & 0 & 3 & 4 & 4 & 4 & 4 & $\mathrm{M}$ \\
\hline At $(\%)^{(3)}$ & 100 & 16,1 & 93,5 & 96,7 & 38,7 & 100 & 77,4 & 3,2 & 12,9 & 96,7 & 30,0 & 56,6 & 80,6 & 83,3 & 100 \\
\hline
\end{tabular}

${ }^{(1)}$ ZC: zona de conforto; ARP: altura em relação à plataforma; CRSIP: comprimento em relação ao ponto de indexação do assento (SIP); LA: largura da almofada; LE: largura do encosto; CE: comprimento do encosto; AL: ajuste longitudinal; AVPM: ajuste vertical a partir da posição média; RIE: regulagem da inclinação do encosto; RP: regulagem de pressão; APD: altura do primeiro degrau; PD: profundidade do degrau; LD: largura do degrau; DVUDS: distância vertical do último degrau até a soleira; EED: espaçamento entre degraus. ${ }^{(2)}$ Monodegrau. ${ }^{(3)}$ Atendimento às conformidades de cada critério. 
amostrados, em relação à norma ISO 15077 (ISO, 1996), e o freio de estacionamento e o pedal de acelerador foram os comandos que menos atenderam à referida norma; entre os raramente utilizados, o comando de acionamento da tração dianteira foi o que melhor atendeu à norma ISO 15077 (ISO, 1996), e o comando chave de luzes foi o que menos atendeu à referida norma; entre os comandos de controle do sistema hidráulico, o comando de posição atendeu à norma ISO 15077 (ISO, 1996) com maior expressão, e o comando de controle remoto foi o que menos atendeu à mesma norma; entre os comandos de controle do câmbio, o seletor de marchas foi o que melhor atendeu à norma ISO 15077 (ISO, 1996), e o seletor de regime foi o que menos atendeu à referida norma; dos comandos acionados exclusivamente pelos pés, o pedal de freio esquerdo foi o que melhor atendeu à norma ISO 15077 (ISO, 1996), considerandose as suas ocorrências nas áreas de alcance em relação ao SIP; o pedal de embreagem foi o comando que melhor atendeu à norma ISO 4253 (ISO, 1993) e o pedal do acelerador foi o que esteve em maior desconformidade com a mesma norma, considerando-se as suas ocorrências nas áreas de alcance em relação ao SIP.

Quanto aos acessos, tratores com potência bruta do motor na faixa de 75 a $100 \mathrm{~kW}$ foram os que melhor atenderam à norma ISO 4253 (ISO, 1993).

Em relação aos assentos, a maioria dos que equipam os tratores agrícolas não atenderam à norma ISO 4253 (ISO, 1993) em relação aos requisitos regulagem do encosto do assento, regulagem no plano horizontal e na altura do assento relativa à plataforma de apoio para os pés.

\section{Conclusões}

1. As marcas de tratores agrícolas nacionais apresentam a mesma prioridade no atendimento aos requisitos de projeto de ergonomia e segurança em postos de operação.

2. $\mathrm{O}$ assento representa a prioridade de maior ordem, seguido dos comandos, e em última posição estão os acessos ao posto de operação.

3. O comprimento do encosto do assento e o espaçamento entre degraus são considerados critérios prioritários nos projetos de todos os postos de operação, e o ajuste vertical da posição média do assento tem a menor prioridade.

\section{Referências}

ABNT. Associação Brasileira de Normas Técnicas. NBR ISO 4252: Tratores agrícolas: local de trabalho do operador, acesso e saída: dimensões. Rio de Janeiro, 2000. 4p.

ABNT. Associação Brasileira de Normas Técnicas. NBR ISO 4254-1: Tratores e máquinas agrícolas e florestais: recursos técnicos para garantir a segurança: parte 1: geral. Rio de Janeiro, 1999a. 13p.

ABNT. Associação Brasileira de Normas Técnicas. NBR NM-ISO 5353: Máquinas rodoviárias, tratores e máquinas agrícolas e florestais: ponto de referência do assento. Rio de Janeiro, 1999b. 5p.

COUTO, H.A. Ergonomia aplicada ao trabalho: manual técnico da máquina humana. Belo Horizonte: Ergo, 1996. v.2. 383p.

CSILLAG, J.M. Análise do valor. 4.ed. São Paulo: Atlas, 1995. $357 \mathrm{p}$.

DEBIASI, H. Diagnósticos dos acidentes de trabalho e das condições de segurança na operação de conjuntos tratorizados. 2003. 291p. Dissertação (Mestrado) - Universidade Federal de Santa Maria, Santa Maria.

DEBIASI, H.; SCHLOSSER, J.F.; PINHEIRO, E.D. Características ergonômicas dos tratores agrícolas utilizados na Região Central do Rio Grande do Sul. Ciência Rural, v.34, p.1807-1811, 2004.

FONTANA, G.; SILVA, R.P.; LOPES, A.; FURLANI, C.E.A. Avaliação de características ergonômicas no posto do operador em colhedoras combinadas. Engenharia Agrícola, v.24, p.684-694, 2004.

GRANDJEAN, E. Manual de ergonomia: adaptando o trabalho ao homem. 4.ed. Porto Alegre: Artes Médicas, 1998. 338p.

ISO. International Organization for Standardization. ISO 15077: tractors and machinery for agriculture and forestry: operator controls: actuating forces, their displacement and location. Genève, 1996.

ISO. International Organization for Standardization. ISO 4252 : agricultural tractors: access, exit and operator's workplace: dimensions. Genève, 1992. 6p.

ISO. International Organization for Standardization. ISO 4253: agricultural tractors: operator's seating accommodation: dimensions. Genève, 1993. 4p.

ISO. International Organization for Standardization. ISO 4254-1: agricultural machinery: safety: part 1: general requirements. Genève, 2008.

KUMAR, A.; MOHAN, D.; MAHAJAN, P. Studies on tractor related injuries in Northern India. Accident Analysis and Prevention, v.30, p.53-60, 1998.

LIMA, J.S.S.; SOUZA, A.P.; MACHADO, C.C.; OLIVEIRA, R.B. Avaliação de alguns fatores ergonômicos nos tratores "feller-buncher" e "skidder" utilizados na colheita de madeira. Revista Árvore, v.29, p.291-298, 2005.

MÁRQUEZ, L. Solo tractor’90. Madrid: Laboreo, 1990. 231p. MEHTA, C.R.; TEWARI, V.K. Seating discomfort for tractor operators: a critical review. International Journal of Industrial Ergonomics, v.25, p.661-674, 2000. 
ROBIN, P. Segurança e ergonomia em maquinaria agrícola: tratores agrícolas. São Paulo: Fundacentro, 1987. 22p.

ROMANO, L.N. Modelo de referência para o processo de desenvolvimento de máquinas agrícolas. 2003. 266p. Tese (Doutorado) - Universidade Federal de Santa Catarina, Florianópolis.

ROZIN, D. Conformidade do posto de operação de tratores agrícolas nacionais com normas de ergonomia e segurança. 2004. 187p. Dissertação (Mestrado) - Universidade Federal de Santa Maria, Santa Maria.

SANTOS FILHO, P.F. dos; FERNANDES, H.C.; QUEIROZ, D.M. de; SOUZA, A.P.; CAMILO, A.J. Avaliação dos níveis de vibração vertical no assento de um trator agrícola de pneus utilizando um sistema de aquisição automática de dados. Revista Árvore, v.27, p.887-895, 2003.

SCHLOSSER, J.F.; DEBIASI, H. Caracterização dos acidentes com tratores agrícolas. Ciência Rural, v.32, p.977-981, 2002.

SCHLOSSER, J.F.; DEBIASI, H.; PARCIANELLO, G.; RAMBO, L. Antropometria aplicada aos operadores de tratores agrícolas. Ciência Rural, v.32, p.983-988, 2002.

VALENT, F.; SCHIAVA, F.; SAVONITTO, C.; GALLO, T.; BRUSAFERRO, S.; BARBONE, F. Risk factors for fatal road traffic accident in Udine, Italy. Accident Analysis and Prevention, v.34, p.71-84, 2002.

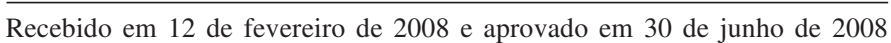

